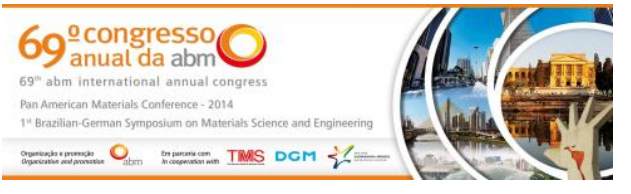

Tema: Materiais com efeito de memória de forma

\title{
INFLUÊNCIA DO TRATAMENTO TÉRMICO NAS TEMPERATURAS DE TRANSFORMAÇÃO DE LIGA Ni-Ti-Cu SOLIDIFICADA RAPIDAMENTE COM EFEITO DE MEMÓRIA DE FORMA*
}

\author{
Alana Pereira Ramos ${ }^{1}$ \\ George Carlos dos Santos Anselmo ${ }^{1}$ \\ Walman Benicio de Castro ${ }^{2}$
}

\section{Resumo}

O efeito de memória de forma tem como principal objetivo a recuperação da forma por meio de aquecimento acima das temperaturas críticas de transformação. Fitas da liga $\mathrm{Ti}_{43,5} \mathrm{Cu}_{37,8} \mathrm{Ni}_{18,7}$ foram preparadas através do processo melt spinnig com velocidade da roda de $21 \mathrm{~m} / \mathrm{s}$ e $63 \mathrm{~m} / \mathrm{s}$. As fitas foram tratadas termicamente numa temperatura de $700^{\circ} \mathrm{C}$ por 15 minutos em forno e resfriadas ao ar. A caracterização das fitas foi feita por calorimetria diferencial de varredura (DSC) e análise por difração de raios $X(D R X)$. Foi observado que com uma menor velocidade de rotação da roda $(21 \mathrm{~m} / \mathrm{s})$ a fita apresentou-se totalmente cristalina, e quando aumentou-se a velocidade linear da roda para $63 \mathrm{~m} / \mathrm{s}$ a fita apresentou-se completamente amorfa. Após o tratamento térmico todas as fitas apresentaram as transformações martensíticas e auteníticas. Observou-se que a velocidade da roda tem grande influência nas temperaturas de transformação, chegando à variação de aproximadamente $7^{\circ} \mathrm{C}$. O tratamento térmico remove todos os defeitos produzidos pelo processo de solidificação rápida e produz um rápido crescimento de grão, favorecendo ao aumento das temperaturas de transformação.

Palavras chave: Ligas Ti-Ni-Cu; Memória de forma; Solidificação rápida.

\section{THE EFFECT OF THE HEAT TREATMENT ON TRANSFORMATION TEMPERATURES IN RAPIDLY SOLIDIFIED Ti-Ni-Cu SHAPE MEMORY ALLOY}

\begin{abstract}
The shape memory effect (SME) is strictly related to the reversible martensitic transformation. Shape memory characteristics of Ti-37,8Cu-18,7Ni alloy ribbons prepared by melt spinning were investigated by means of differential scanning calorimetry (DSC) and X-ray diffraction. In these experiments particular attention has been paid to change of the velocity of cooling wheel from 21 to $63 \mathrm{~m} / \mathrm{s}$. Then the cooling rates of ribbons were controlled. The effect of this cooling rate on austenitic and martensitic transformations behaviors is discussed.the ribbon is produced at 63 $\mathrm{m} / \mathrm{s}$ the amorphous structure without any crystal phases was observed. The ribbons of this alloy was subjected to annealing to a temperature of $700^{\circ} \mathrm{C}$ for 15 minutes. Thermal treatment removes all defects produced by rapid solidification process and produces a rapid grain growth and the increase of transformation temperatures.

Keywords: Ni-Ti-Cu alloy; Shape memory; Rapid solidification; Melt Spinning.

1 Alunos da Pós-graduação em Ciência e Engenharia de Materiais, UFCG, Campina Grande, PB. Brasil.

2 Engenheiro mecânico, Professor, CCT, Unidade Acadêmica de Engenharia Mecânica, UFCG, Campina Grande, PB, Brasil; walman@dem.ufcg.edu.br.
\end{abstract}

* Contribuição técnica ao $69^{\circ}$ Congresso Anual da ABM - Internacional e ao 14ํㅡㄹ ENEMET - Encontro Nacional de Estudantes de Engenharia Metalúrgica, de Materiais e de Minas, 21 a 25 de julho de 2014, São Paulo, SP, Brasil. 


\section{INTRODUÇÃO}

Os termossensores são largamente usados em aplicações domésticas e em equipamentos industriais para controle de temperatura. Em muitas aplicações, especialmente em sistema de alarme contra incêndio e em dispositivos de proteção contra superaquecimento, é muito importante aumentar a velocidade de resposta, e para isso é necessário miniaturizar esses dispositivos com garantia de baixo custo [1].

O material mais promissor para atender tais exigências são as ligas metálicas com Efeito de Memória de Forma (EMF). A designação de ligas com memória de forma é aplicada a um grupo de materiais metálico que demonstra a capacidade de retomar uma forma ou tamanho previamente definido quando sujeitas a um ciclo térmico apropriado [2].

Em virtude de possuírem propriedades específicas, as ligas com efeito de memória de forma (SMA's) são utilizadas em muitas áreas da ciência e tecnologia e está cada vez mais aumentando o campo de suas aplicações. As aplicações mais importantes são na engenharia elétrica e mecânica, tecnologia aeroespacial, medicina e robótica. Com o surgimento de novos desafios na engenharia, novas pesquisas e desenvolvimentos em ligas com efeito de memória de forma com espessuras finas se fazem necessárias, para que atenda as necessidades de miniaturização, operação mais econômica e resposta rápida de dispositivos. Uma perspectiva consiste em produzir fitas com espessura de $100 \mu \mathrm{m}$ ou menos a partir de ligas à base de nitinol (NiTi). Porém, é muito difícil produzir fitas com essa espessura a partir de ligas pré-deformadas à base de NiTi utilizando a técnica convencional, que é a de laminação, pois o trabalho demanda um tempo maior e o custo do processo se torna alto. Além disso, a tecnologia convencional por laminação de obtenção de fitas provoca diminuição do efeito de memória de forma das ligas [3].

Diante disso, é bem sugestivo um projeto onde se possam obter fitas de ligas com efeito de memória de forma utilizando resfriamento rápido (técnica de melt spinning). Essa técnica permitirá obtenção de uma grande quantidade de fitas de novas ligas com memória de forma com variação nas propriedades físicas e mecânicas. A principal vantagem dessa nova tecnologia está no fato de que ela permite, através do resfriamento rápido, a obtenção de ligas com estrutura amorfa ou parcialmente amorfas, que depois do tratamento térmico, se tornam estruturas micro cristalinas ou nano cristalinas com um fino grau de dispersão da ordem 1-2, o que não é possível obter através da tecnologia convencional de processamento de ligas com efeito de memória de forma [4].

Assim o objetivo desse trabalho foi caracterizar fitas da Ti-37,8Cu-18,7Ni (\% em peso) com memória de forma, obtidas pelo processo de Melt Spinning, com a velocidade linear da roda de $21 \mathrm{~m} / \mathrm{s}$ e $63 \mathrm{~m} / \mathrm{s}$.

\section{MATERIAIS E MÉTODOS}

A liga bruta de fusão de Ti-37,8Cu-18,7Ni (\%at) foi produzida pelo processo de fusão em forno a arco. As fitas foram obtidas através da técnica de melt spinning, Pedaços do lingote foram previamente preparados e colocados no interior de um cadinho de quartzo e estes alojados no interior de uma bobina de indução que se encontra numa câmara com atmosfera de argônio para então ser refundido. A ejeção da liga líquida ocorreu na temperatura de aproximadamente $1350^{\circ} \mathrm{C}$, com uma pressão de

\footnotetext{
* Contribuição técnica ao $69^{\circ}$ Congresso Anual da ABM - Internacional e ao 14ํㅡㄹ ENEMET - Encontro Nacional de Estudantes de Engenharia Metalúrgica, de Materiais e de Minas, 21 a 25 de julho de 2014, São Paulo, SP, Brasil.
} 


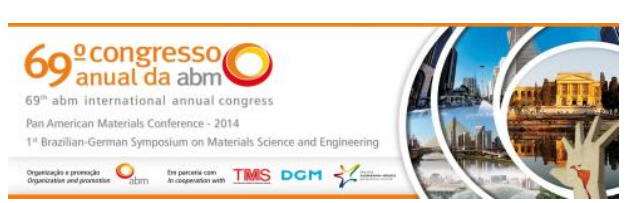

austenítica e martensítica, já a amostra que solidificou com uma velocidade linear da roda de $63 \mathrm{~m} / \mathrm{s}$ não apresentou temperaturas de transformação, austenítica e martensítica, em virtude do seu estado amorfo.

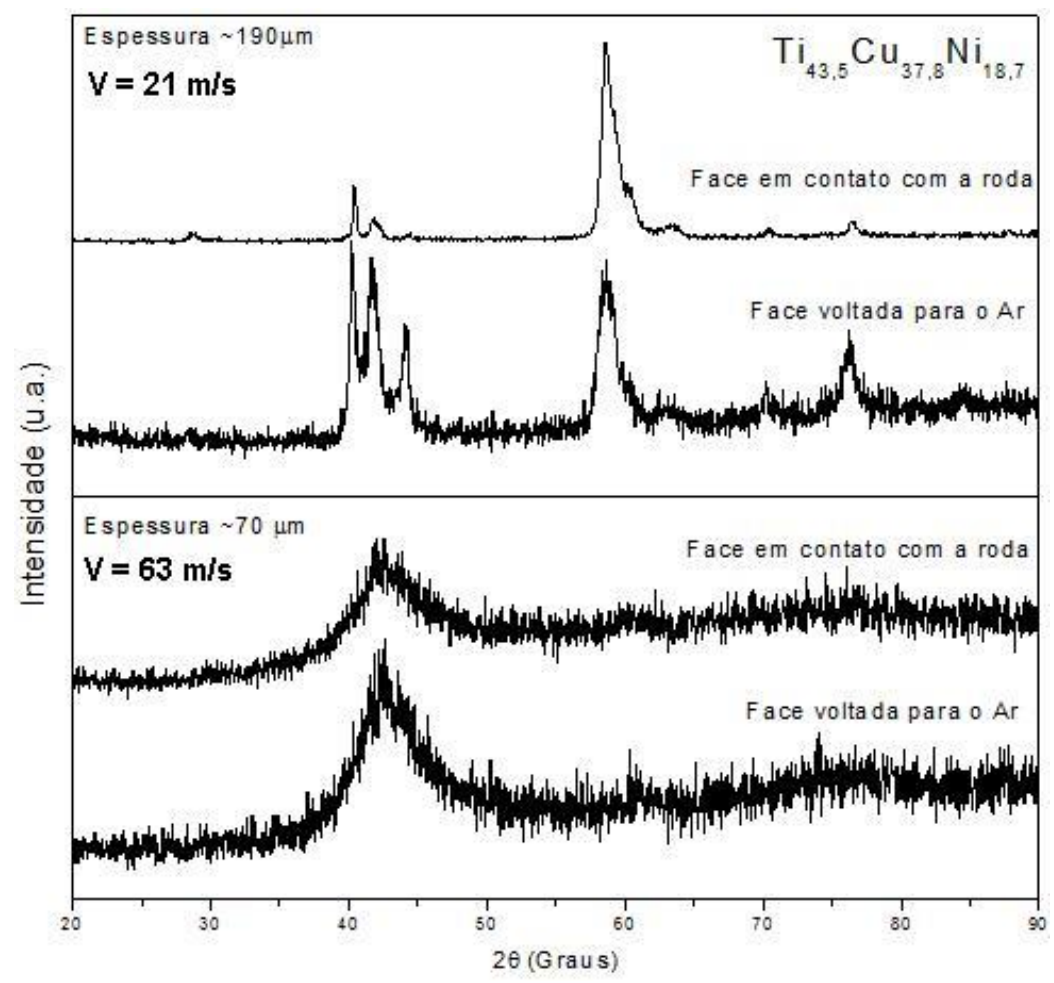

Figura 2. Difração de raios $x$ das fitas não tratadas.

A figura 4 apresenta as curvas de DSC para as fitas tratadas termicamente, onde as mesmas foram aquecidas até $700^{\circ} \mathrm{C}$ por 15 minutos, em seguida foram retiradas do forno e resfriadas ao ar. Observa-se que todas as fitas apresentaram temperatura de transformação austenítica e martensítica. A tabela 1 apresenta os resultados das temperaturas de transformação para as fitas não-tratadas e tratadas termicamente. Analisando as Figuras 3 e 4 e a tab. 1 abaixo observou-se que as temperaturas de transformação aumentaram para as fitas tratadas termicamente. Isso aconteceu, provavelmente, porque o tratamento térmico produz dois efeitos nas fitas [7]: 1) Eliminação dos defeitos internos e da tensão residual, isto porque, o processo de solidificação rápida produzida pela alta extração de calor da roda de cobre quando da obtenção das fitas, gera uma grande quantidade de defeitos e tensão residual [8]; 2) Rápido crescimento dos grãos que antes eram muito finos em virtude do rápido resfriamento [9].

\section{CONCLUSÕES}

A técnica de melt spinning permite a produção de fitas muito finas da ordem de micrometro em apenas uma etapa de processamento. Fitas amorfas sem nenhuma fase cristalina foram produzidas na liga Ti-37,8Cu-18,7 Ni a uma velocidade linear da roda de $63 \mathrm{~m} / \mathrm{s}$. O tratamento térmico das fitas provocou um aumento das temperaturas de transformação austeniticas e martensíticas, pois a temperatura usada no tratamento térmico foi suficiente para remover todos os defeitos produzidos pelo processo de solidificação rápida e produzir um rápido crescimento de grão, favorecendo o aumento das temperaturas de transformação.

* Contribuição técnica ao $69^{\circ}$ Congresso Anual da ABM - Internacional e ao 14ํㅡㄹ ENEMET - Encontro Nacional de Estudantes de Engenharia Metalúrgica, de Materiais e de Minas, 21 a 25 de julho de 2014, São Paulo, SP, Brasil. 

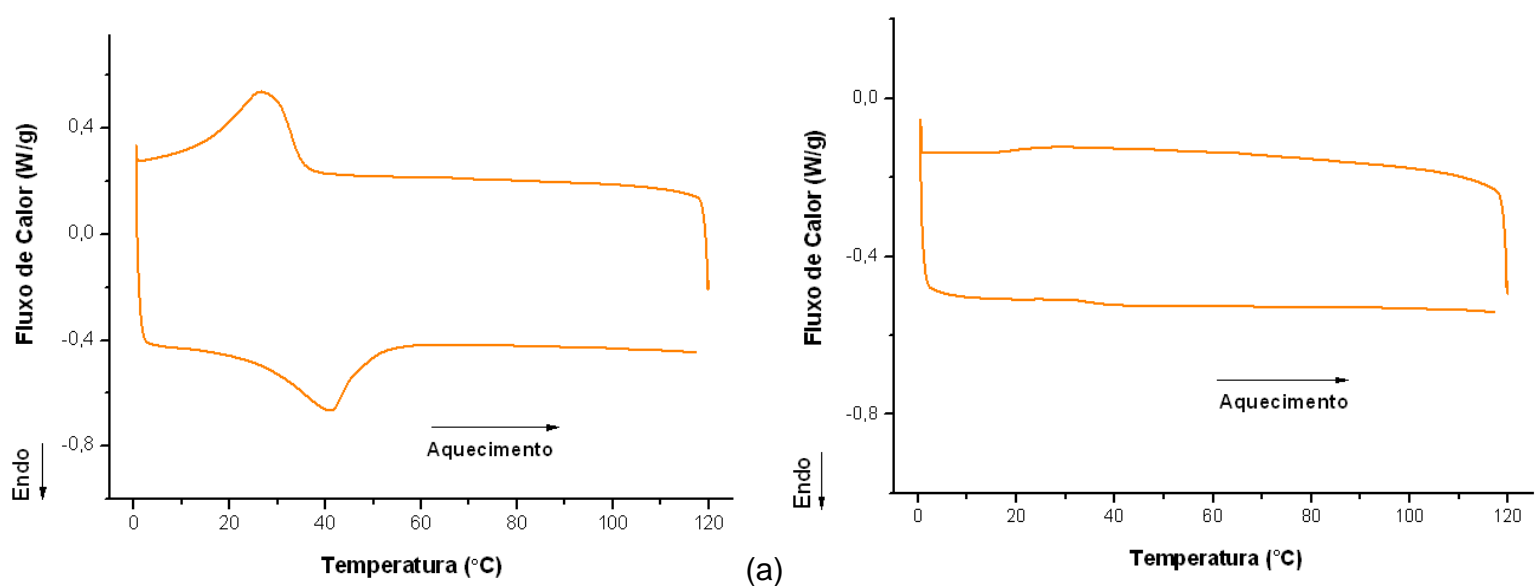

Figura 3. DSC das fitas obtidas por melt spinning sem tratamento térmico: (a) $V=21 \mathrm{~m} / \mathrm{s}$, (b) $V=63 \mathrm{~m} / \mathrm{s}$.
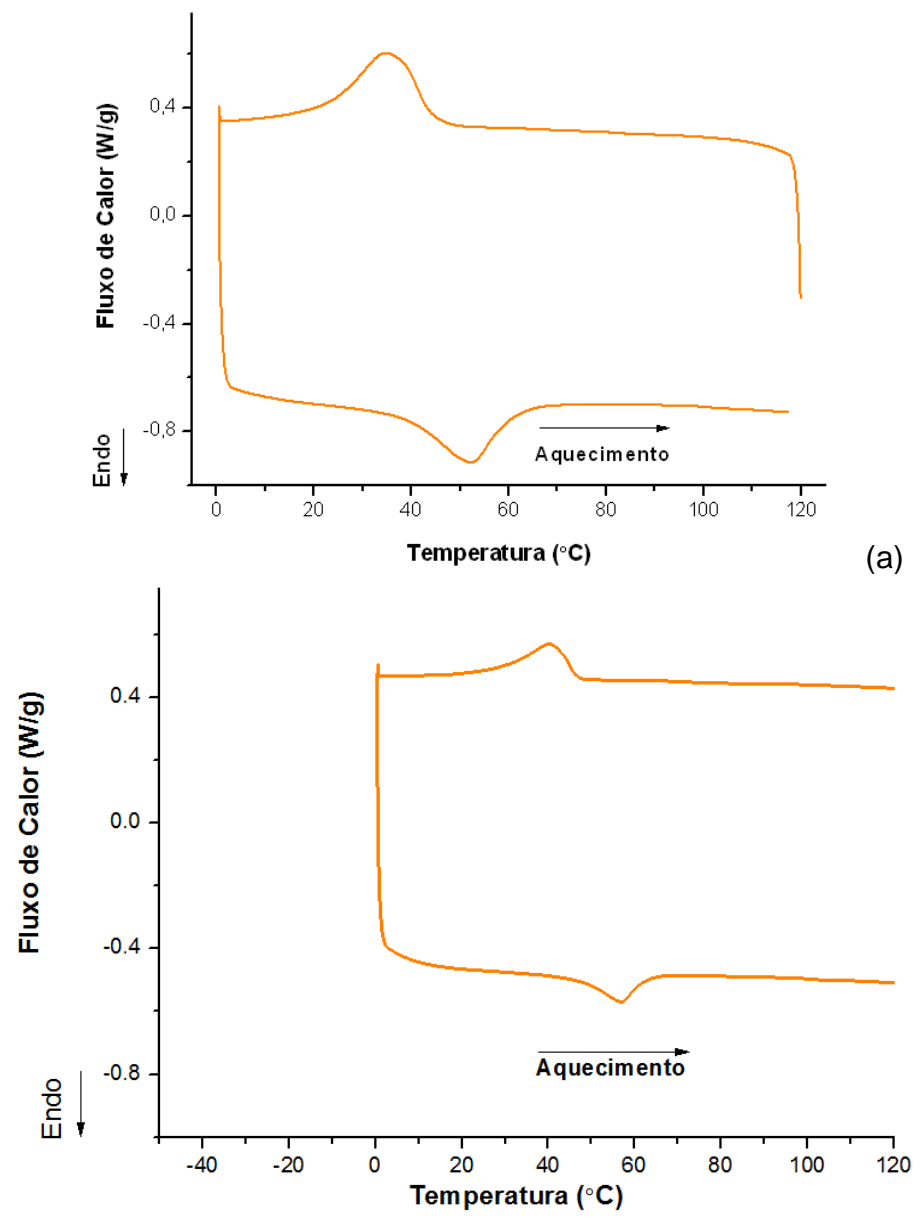

(a)

(b)

Figura 4. DSC das fitas por melt spinning com tratamento térmico: a) $V=21 \mathrm{~m} / \mathrm{s}$, (b) $V=63 \mathrm{~m} / \mathrm{s}$.

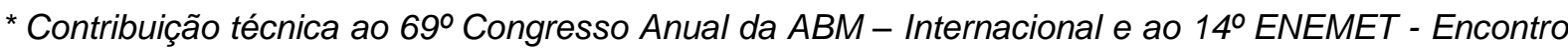
Nacional de Estudantes de Engenharia Metalúrgica, de Materiais e de Minas, 21 a 25 de julho de 2014, São Paulo, SP, Brasil. 
Tabela 1. Valores das temperaturas de transformação.

\begin{tabular}{c|c|c|c|c}
\hline Amostra & $\begin{array}{c}\mathrm{A}_{\mathrm{s}} \\
\left({ }^{\circ} \mathrm{C}\right)\end{array}$ & $\begin{array}{c}\mathrm{A}_{\mathrm{f}} \\
\left({ }^{\circ} \mathrm{C}\right)\end{array}$ & $\begin{array}{c}\mathrm{M}_{\mathrm{s}} \\
\left({ }^{\circ} \mathrm{C}\right)\end{array}$ & $\begin{array}{c}\mathrm{M}_{\mathrm{f}} \\
\left({ }^{\circ} \mathrm{C}\right)\end{array}$ \\
\hline $\begin{array}{c}\text { Sem Tratamento Térmico } \\
\mathrm{V}=21 \mathrm{~m} / \mathrm{s}\end{array}$ & 25,80 & 50,48 & 36,31 & 13,95 \\
\hline $\begin{array}{c}\text { Com Tratamento Térmico } \\
\mathrm{V}=21 \mathrm{~m} / \mathrm{s}\end{array}$ & 39,57 & 61,86 & 46,21 & 23,57 \\
\hline $\begin{array}{c}\text { Sem Tratamento Térmico } \\
\mathrm{V}=62 \mathrm{~m} / \mathrm{s}\end{array}$ & - & - & - & - \\
\hline $\begin{array}{c}\text { Com Tratamento Térmico } \\
\mathrm{V}=62 \mathrm{~m} / \mathrm{s}\end{array}$ & 46,18 & 63,37 & 46,53 & 27,67 \\
\hline
\end{tabular}

\section{REFERÊNCIAS}

1 Bellouard Y. Shape memory alloys for microsystems: A review from a material research perspective. Materials Science and Engineering A. 2008;481-482:582-589.

2 Fernandes FMB. Ligas Com Memória de Forma. Lisboa: Departamento de ciência dos Materiais (CENIMAT), Universidade de Lisboa; 2006.

3 Saburi T. In: Otsuka K., Wayman C.M. (Eds.). Shape Memory Materials. Cambridge: Cambridge University Press; 1998. p. 49-73.

4 Duwez P. Metallic Glasses - Historical Background. Topics in Applied Physics. 1981;46:19-23.

5 Shelyakov, AV, Sitnikov NN, Menushenkov AP, Korneev AA, Rizakhanov RN, Sokolova NA. Fabrication and characterization of amorphous-crystalline TiNiCu melt-spun ribbons. Journal of Alloys and Compounds. 2013;5775:251-254.

6 Chen YZ, Liu F, Yang GC, Liu N, Yang CL, Xie H, Zhou YH. Grain refinement of $\mathrm{Fe}_{75} \mathrm{Ni}_{25}$ alloys at low undercooling. Materials Characterization. 2008;59:412-416.

7 Lin NK, Wu SK. Martensitic transformation of grain-size mixed Ti51Ni49 melt-spun ribbons. Journal of Alloys and Compounds. 2006:171-175.

8 Karaköse E, Keskin M. Morphological characteristic of the conventional and melt-spun Al-10Ni-5.6Cu (in wt.\%) alloy. Materials Characterization. 2009;60:1569-77.

9 Mehrabi K, Bruncko M, KneissI AC. Microstructure, mechanical and functional properties of NiTi-based shape memory ribbons. Journal of Alloys and Compounds. 2012;526:4552.

\footnotetext{
* Contribuição técnica ao 69ำ Congresso Anual da ABM - Internacional e ao 14ํㅡㄹ ENEMET - Encontro Nacional de Estudantes de Engenharia Metalúrgica, de Materiais e de Minas, 21 a 25 de julho de 2014, São Paulo, SP, Brasil.
} 